\title{
Modelagem Matemática e Aprendizagem Significativa: uma Relação Subjacente
}

\author{
Mathematical Modeling and Meaningful Learning: an Underlying Relationship
}

\author{
Jerson Sandro Santos de Souza
}

Secretaria de Estado de Educação do Amazonas, AM, Brasil.

E-mail: jerson.souza@seducam.pro.br

\begin{abstract}
Resumo
O presente artigo propõe-se a apresentar algumas reflexões, embasadas em uma pesquisa bibliográfica, sobre a modelagem matemática como metodologia de ensino. Discutem-se os pressupostos, as características, as etapas e as possibilidades de organização curricular de atividades que são pautadas nessa alternativa metodológica. Destaca-se o papel da teoria da aprendizagem significativa na fundamentação e na defesa da modelagem matemática como proposta de ensino. Efetua-se um confronto das várias perspectivas oriundas dos textos científicos selecionados para estudo e análise, de modo a buscar convergências que evidenciem o potencial da modelagem matemática para: (1) favorecer a utilização pedagógica dos conhecimentos prévios dos aprendizes e (2) instigar o comprometimento deles com as situações de aprendizagem. Concluise o trabalho apresentando argumentos que caracterizam essa metodologia de ensino como favorecedora da aprendizagem significativa de conteúdos matemáticos, com o intuito de disponibilizar aos professores de matemática subsídios teóricos que os auxiliem na tomada de consciência do processo de interação cognitiva, entre as novas informações e os conhecimentos prévios do educando, subjacente às atividades de modelagem matemática.
\end{abstract}

Palavras-chave: Modelagem Matemática. Aprendizagem Significativa. Educação Matemática.

\begin{abstract}
This article presents some reflections based on a bibliographic research on mathematical modeling as a teaching methodology. The assumptions, characteristics, stages, and possibilities of curricular organization of activities based on this methodological alternative are discussed. The role of significant learning theory in the foundation and defense of mathematical modeling as a teaching proposal is highlighted. A comparison of the various perspectives from the scientific texts selected for study and analysis is made to find convergences that highlight the potential of mathematical modeling to: (1) favor the pedagogical use of the students' prior knowledge and (2) instigate their commitment to learning situations. The work is concluded with arguments that characterize this teaching methodology as fostering significant learning of mathematical content, to provide mathematics teachers with theoretical subsidies that help them to become aware of the cognitive interaction process, among the new information and the students' previous knowledge that underlies the mathematical modeling activities.
\end{abstract}

Keywords: Mathematical Modeling. Meaningful Learning. Mathematics Education.

\section{Introdução}

A modelagem matemática é um método de pesquisa de matemática aplicada, utilizado na resolução de problemas concretos de diversas áreas do conhecimento. Os problemas levantados são resolvidos, como pressupõe o método, mediante a construção de modelos matemáticos que simulem os sistemas reais em estudo. Com base numa representação matemática adequada desses sistemas, pode-se prever o comportamento dos fenômenos em questão, usando ideias e técnicas essencialmente matemáticas. A resolução da tradução matemática do problema torna possível, com certa margem de erro, a resolução do problema real. Em resumo: “[...] a modelagem matemática consiste na arte de transformar problemas da realidade em problemas matemáticos e resolvêlos interpretando suas soluções na linguagem do mundo real" (Bassanezi, 2010, p.16).
A busca por um modelo válido, apropriado à situação real em estudo, é o que está basicamente em jogo nesse processo. Se o modelo matemático mostrar-se inadequado, dados e hipóteses devem ser incluídos ou modificados e o processo refeito; isto leva a uma atividade cíclica de análise-reflexãoconstrução.

$\mathrm{O}$ processo de modelagem une a precisão, o rigor e a generalização da matemática ao caráter investigativo, criativo e crítico das abordagens pautadas em situações da vida real. Desse modo, por favorecer uma articulação entre a construção e a aplicação de conceitos, dentro e fora da própria matemática, a modelagem matemática é vista não só como um método ou processo de pesquisa, mas também como uma proposta metodológica para a construção do conhecimento matemático por parte dos educandos.

Entendida como metodologia de ensino, a modelagem pauta-se nos princípios de problematização e investigação 
(Barbosa, 2001, 2004). Ou seja, ela privilegia atividades nas quais os alunos são convidados a levantar questões acerca de determinado tema, bem como a buscar, selecionar e organizar informações que contribuam para a solução do problema colocado. Nesse sentido, a "Modelagem é um processo muito rico de encarar situações e culmina com a solução efetiva do problema real e não com a simples resolução formal de um problema artificial" (D’Ambrósio, 1986, p.11).

Além disso, as atividades organizadas, segundo os pressupostos da modelagem matemática, podem ser qualificadas como potencialmente significativas, uma vez que fomentam ambientes de aprendizagem nos quais os alunos são levados a compreender significativamente as ideias que são capazes de verbalizar. Quer dizer, na medida em que essa alternativa metodológica aumenta o interesse dos aprendizes e possibilita uma compreensão crítica dos conteúdos mediante discussões e reflexões realizadas em sala de aula, ela oportuniza a aprendizagem significativa dos conteúdos matemáticos abordados.

Segundo Ausubel etal.(1980), a aprendizagem significativa ocorre quando as ideias expressas simbolicamente nos materiais instrucionais interagem de maneira não-arbitrária e não-literal com os conhecimentos prévios do sujeito que aprende, resultando na emergência de significados claros, precisos, diferenciados e transferíveis. Diferentemente, naquilo que os autores chamam aprendizagem mecânica, ocorrem associações arbitrárias e o produto desse tipo de aprendizagem é, geralmente, de duração, utilidade e significados transitórios. Fica evidente, portanto, que a educação escolar deve preocupar-se com o favorecimento da aprendizagem significativa.

Assim sendo, neste trabalho, tecemos, com base em uma pesquisa bibliográfica, algumas reflexões sobre como a modelagem pode contribuir para o ensino da matemática, especialmente no que toca à sua capacidade de favorecer a aprendizagem significativa de conteúdos matemáticos.

\section{Metodologia}

Optou-se pela realização de uma pesquisa bibliográfica, pois esse tipo de metodologia permite "[...] ao investigador a cobertura de uma gama de fenômenos muito mais ampla do que aquela que poderia pesquisar diretamente" (Gil, 2008, p.50). Essa característica torna a pesquisa bibliográfica ideal para os fins deste artigo, visto que a modelagem matemática assume, enquanto elemento didático-metodológico, inúmeras facetas, muito difíceis de serem contempladas diretamente.

A pesquisa bibliográfica é uma modalidade de estudo e análise de documentos decorrentes de fontes científicas, como livros, monografias, dissertações, teses e artigos científicos. Ela pauta-se, portanto, na contribuição de diferentes autores sobre o tema. Entretanto, mesmo que os documentos já tenham recebido tratamento analítico, "[...] a pesquisa bibliográfica não é mera repetição do que já foi dito ou escrito sobre certo assunto, mas propicia o exame de um tema sob novo enfoque ou abordagem, chegando a conclusões inovadoras" (Marconi \& Lakatos, 2003, p.183). De modo geral, a pesquisa bibliográfica implica um "[...] conjunto ordenado de procedimentos de busca por soluções, atento ao objeto de estudo, e que, por isso, não pode ser aleatório" (Lima \& Mioto, 2007, p.38).

Considerando os pressupostos mencionados, realizaramse, inicialmente, a seleção e a análise de diferentes textos científicos que apresentassem discussões de natureza psicológica, histórico-epistemológica e didática sobre o fenômeno da modelagem matemática como metodologia de ensino. Em um segundo momento, com base nas leituras, efetuou-se um confronto das várias perspectivas oriundas dos textos, buscando-se convergências que evidenciassem o potencial da modelagem matemática para: (1) favorecer a utilização pedagógica dos conhecimentos prévios dos aprendizes e (2) instigar o comprometimento deles com as situações de aprendizagem. Esses dois parâmetros funcionaram como elementos norteadores da pesquisa, pois, além de propiciarem uma organização do ensino que torna o saber acessível e desejável aos educandos, ainda oferecerem evidências de uma aprendizagem significativa.

\section{Modelagem Matemática: um Contraponto ao Ensino Tradicional de Matemática}

A modelagem matemática, na perspectiva da Educação Matemática, propõe que as situações didáticas sejam organizadas a partir de problemas reais e interessantes para os aprendizes, levando em consideração seus conhecimentos anteriores, no intuito de promover uma articulação entre a construção e a aplicação de conceitos, dentro e fora da própria matemática. Nesse ambiente de aprendizagem, temos a oportunidade de despertar o interesse dos educandos e favorecer a construção de um conhecimento mais crítico e reflexivo dos conteúdos da matemática (Almeida \& Dias, 2004).

Tal perspectiva contrapõe-se ao método tradicional de ensino da matemática, que privilegia a resolução de exercícios e problemas típicos dentro de contextos previsíveis e compartimentados. $\mathrm{Na}$ abordagem tradicional, o professor de matemática "[...] apresenta o conteúdo oralmente, parte de definições e exemplos, segue com exercícios de fixação, e imagina-se que o aluno aprendeu pela reprodução" (Santos \& Bisognin, 2007, p.101).

Segundo Barbosa (2001, p. 6), a "Modelagem é um ambiente de aprendizagem no qual os alunos são convidados a indagar e/ou investigar, por meio da matemática, situações oriundas de outras áreas da realidade". Para Biembengut e Hein (2014), os objetivos da modelagem matemática como metodologia de ensino da matemática são: 1) aproximar a matemática de outras áreas de conhecimento; 2) enfatizar a importância da matemática para a formação do aluno; 
3) despertar o interesse pela matemática em vista de sua aplicabilidade; 4) melhorar a apreensão dos conceitos matemáticos; 5) desenvolver a habilidade para resolver problemas; e 6) estimular a criatividade.

É importante destacar que os trabalhos de modelagem matemática repousam sobre os princípios de problematização e investigação (Barbosa, 2001, 2004). Por esse motivo, os alunos são divididos em grupos e a atividade inicia-se com a escolha de um tema. A partir do estudo desse tema, é formulado um problema com referência na realidade que deve sintetizar os diversos interesses das equipes.

O papel do professor é redefinido. Ele deixa de ser o centro do processo de ensino e aprendizagem e passa a atuar como um mediador, fazendo uma ponte entre as disposições individuais e socioculturais do aluno e o objeto de conhecimento. No contexto das atividades, o professor orienta os grupos na transformação de um tema abrangente em uma questão clara, precisa e suscetível de solução, além de contribuir para a construção de argumentos matemáticos que conduzam às soluções dos problemas formulados.

O ambiente de Modelagem está associado à problematização e investigação. $\mathrm{O}$ primeiro refere-se ao ato de criar perguntas e/ou problemas enquanto o segundo à busca, seleção, organização e manipulação de informações e reflexão sobre elas. Ambas atividades não são separadas, mas articuladas no processo de envolvimento dos alunos para abordar a atividade proposta. Nela, podem-se levantar questões e realizar investigações que atinge o âmbito do conhecimento reflexivo. (Barbosa, 2004, p.3).

Além dos princípios de problematização e investigação, podemos salientar o caráter potencialmente significativo das atividades de modelagem matemática. Ele diz respeito à capacidade que essas atividades têm de favorecer a interação entre o conhecimento prévio dos alunos, a motivação para aprender e as novas informações a serem assimiladas. Desse modo, a aprendizagem dos conteúdos matemáticos em jogo segue a curva do interesse real dos aprendizes.

Por fim, à luz do exposto, é fácil oferecer uma forte justificativa para a utilização da modelagem matemática como metodologia de ensino da matemática. Embora a resolução de problemas padronizados e artificiais possa fornecer algum treinamento matemático aos alunos, essa abordagem não possibilita uma compreensão crítica dos conteúdos contemplados pelo ensino. Isso ocorre porque esse tipo de compreensão só pode ser alcançado em contextos que exijam ir além da própria matemática.

Pensemos na seguinte questão: Para que serve a matemática? É impossível respondê-la satisfatoriamente sem recorrer a contextos extramatemáticos; sem recorrer às relações que a matemática estabelece com as outras disciplinas; sem considerar a relevância social da matemática e sem discutir os objetivos maiores da educação. Tal fato torna necessário trabalhar com problemas baseados nos interesses e necessidades dos indivíduos na sociedade, uma vez que essas situações problemáticas propiciam um maior esclarecimento sobre a realidade e sobre o papel que a matemática exerce no mundo e isto contribui para que os aprendizes vejam sentido nos conteúdos matemáticos que estão estudando.

Carvalho (1994, p.88) elabora a situação acima da seguinte forma: "O desafio é ensinar Matemática útil e relevante para o cidadão, sem perder as especificidades e a estrutura inatas à Matemática". É neste sentido "[...] que a aproximação dessas duas forças, aprendizagem significativa e modelagem matemática, contribui para o estabelecimento de uma educação menos impessoal, valorizando o processo de ensino e aprendizagem no sentido da Educação Matemática" (Borssoi \& Almeida, 2004, p.118).

Nessa perspectiva, a abordagem tradicional mostra-se insuficiente. Niss (2012) ressalta que ter bons conhecimentos e habilidades em matemática não é garantia de sucesso em contextos de modelagem. Para tanto, os alunos precisam de um ensino específico, que os instrumentalize para que saibam lidar com problemas nos quais terão de articular conceitos e procedimentos matemáticos e não matemáticos e aplicá-los a situações novas e inesperadas. Eis a importância de um ensino organizado segundo os pressupostos da modelagem matemática.

\section{Modelo Matemático}

A modelagem matemática é um processo que envolve a obtenção de um modelo matemático, que é uma tradução simplificada de uma parcela da realidade. Um modelo matemático pode ser encarado como uma ferramenta que nos permite compreender o passado, o presente e, principalmente, o futuro de determinado fenômeno. Esses modelos são aproximações convenientes de uma realidade pesquisada; por isso, nunca concluem uma verdade definitiva, estando permanentemente sujeitos a modificações. Bassanezi (2010, p.20) define modelo matemático "[...] como um conjunto de símbolos e relações matemáticas que representam de alguma forma o objeto estudado".

Para elaborar os modelos matemáticos, faz-se, comumente, uma análise do sistema real em estudo, a fim de selecionar argumentos e parâmetros tomados como essenciais. Essas informações subsidiarão a construção das hipóteses, que serão representadas por um conjunto de símbolos e equações.

De acordo com Biembengut e Hein (2014, p.12) "Um modelo matemático pode ser formulado em termos familiares, utilizando-se expressões numéricas ou fórmulas, diagramas, gráficos ou representações geométricas, equações algébricas, tabelas, programas computacionais etc.". Consideram que o trabalho com modelos matemáticos é uma arte, “[...] ao formular, resolver e elaborar expressões que valham não apenas para uma solução particular, mas que também sirvam, posteriormente, como suporte para outras aplicações e teorias" (p.13). Os modelos matemáticos constituem, portanto, as formas de representação da realidade cuja obtenção, aplicação e avaliação compõem a modelagem matemática.

Os modelos matemáticos, construídos em atividades de 
modelagem em sala de aula, proporcionam um novo olhar sobre os conceitos matemáticos. Ao mesmo tempo em que o aluno se envolve com questões formais de determinado conteúdo, ele vivencia uma aplicação. Os conteúdos matemáticos adquirem significados. Embora possam ser simples, tais modelos são produtos de um processo de investigação, de criatividade, de tomada de decisões. Em atividades de modelagem, o aprendiz deixa de ser um mero espectador para assumir a condição de sujeito ativo no processo de construção dos seus conhecimentos matemáticos. Daí o principal valor pedagógico dessa proposta de ensino.

\section{Etapas Para o Desenvolvimento de Trabalhos de Modelagem Matemática}

Burak (2004) destaca cinco etapas para o desenvolvimento do trabalho com modelagem matemática na sala de aula, são elas: 1) escolha do tema; 2) pesquisa exploratória; 3 ) levantamento dos problemas; 4) resolução dos problemas e o desenvolvimento da matemática relacionada ao tema; e 5) análise crítica das soluções.

Escolha do tema. Essa escolha parte do interesse dos grupos envolvidos. O tema pode ser apresentado pelo professor ou ser sugerido pelos próprios alunos. Como podem ser quaisquer temas, inicialmente podem não apresentar uma conexão imediata com a matemática. Nesse momento, o professor deve entrar em cena, fazendo uma ponte entre o conhecimento prévio dos alunos e o saber matemático historicamente constituído, a fim de revelar a matemática implícita na situação.

Pesquisa exploratória. Depois da escolha do tema, os alunos devem ser orientados no sentido de construírem um referencial teórico. A pesquisa, que pode ser bibliográfica ou um trabalho de campo, deve contemplar as várias dimensões que compõem a realidade investigada: a dimensão social, econômica, política etc. Entende-se que, para intervir adequadamente na realidade em estudo, o aprendiz deve primeiro conhecê-la.

Levantamento dos problemas. Os dados coletados na pesquisa exploratória respaldarão a formulação dos problemas. Os problemas de modelagem matemática são diferentes daqueles apresentados nos livros didáticos, pois eles têm referência na realidade e no interesse dos grupos. Nesta etapa, o professor incentiva os alunos a conjecturarem sobre tudo o que pode ter uma relação com a matemática, a construírem hipóteses, a analisarem as situações e a justificarem suas ações. São os problemas levantados que determinam os conteúdos a serem trabalhados. Tal característica é contrária aos princípios do método tradicional, que enfatiza o cumprimento de um programa linear e logicamente ordenado.

Resolução dos problemas e o desenvolvimento da matemática relacionada ao tema. Nesta etapa, faz-se uso de todo ferramental matemático disponível para a resolução dos problemas levantados. Pode-se, inicialmente, utilizar procedimentos empíricos para alcançar os primeiros resultados e aproximações e, na sequência, desenvolver de forma analítica os conteúdos matemáticos, formalizando-os. É nesta etapa que acontece a construção dos modelos matemáticos que, mesmo simples, oportunizam momentos ricos para o desenvolvimento do raciocínio lógico-matemático. Os conteúdos matemáticos ganham importância e significado. Durante a resolução do problema, o aprendiz pode precisar de conceitos matemáticos com os quais ainda não trabalhou, cabendo ao professor auxiliá-lo na construção desse conhecimento.

Análise crítica das soluções. É o momento no qual a validação do modelo matemático está em jogo e é marcado pela criticidade e reflexão acerca dos resultados obtidos. Nesta fase, os aprendizes justificam os procedimentos adotados, analisam e discutem as soluções encontradas, enfim, é um momento de socialização de ideias e de interação entre os grupos. Esta fase possibilita o aprofundamento dos conhecimentos matemáticos e não matemáticos inerentes ao tema, bem como a análise da consistência lógica dos argumentos matemáticos utilizados e da sua viabilidade para a situação em estudo.

\section{Modelagem Matemática e Currículo}

No ensino tradicional de matemática, as situaçõesproblema, trazidas para discussão, são idealizadas, fictícias, cuja resolução está diretamente relacionada ao assunto, previamente abordado na exposição do professor. No entanto, uma proposta de modelagem foge a esse paradigma: os problemas levantados é que determinam os conteúdos a serem abordados, o que geralmente leva a contextos desconhecidos, que podem exigir mais tempo para o desenvolvimento das atividades.

Quer dizer, a modelagem matemática como metodologia de ensino pressupõe uma visão mais flexível da organização curricular e não toma como natural a noção de que há uma sequência rígida de conteúdos que deve ser obrigatoriamente cumprida. Essa metodologia parte da premissa de que o interesse do aluno na escolha de um tema conduz a uma motivação, sendo que este interesse gerado nas atividades e os conhecimentos prévios do aprendiz deveriam ser partes fundamentais do currículo. Desse modo, fica subentendido que a modelagem toma como ideal um currículo pautado nos princípios da aprendizagem significativa. Segundo Ausubel et al. (1980, p.294), “Ao planejar um novo currículo ou segmento de um programa de ensino, é importante ter sempre em mente que 'a coisa mais importante que influencia a aprendizagem é o que o aluno já sabe",.

Burak e Barbieri (2005) acrescentam que a aprendizagem significativa permeia as atividades de modelagem matemática porque os conteúdos matemáticos são levados para dentro da vida dos educandos, sendo trabalhados numa perspectiva em que não se segue a ordem lógica dos materiais instrucionais, mas a ordem psicológica, sempre relacionando as novas informações a conceitos já existentes na estrutura de conhecimentos do aluno.

A transição do ensino tradicional para o trabalho com 
modelagem matemática não é uma mudança tão simples. Envolve a adoção de uma postura epistemológica diferenciada ante o processo de ensino e de aprendizagem. Não “[...] é de se esperar que esta mudança ocorra instantaneamente a partir da percepção da plausibilidade da Modelagem no ensino, sob pena de ser abortada no processo" (Barbosa, 2001, p.8).

Em geral, a literatura especializada costuma apresentar cinco argumentos a favor da inclusão da modelagem no currículo: 1) motivação; 2) facilitação da aprendizagem; 3) preparação para utilizar a matemática em diferentes áreas; 4) desenvolvimento de habilidades gerais de exploração; e 5) compreensão do papel sociocultural da matemática (Barbosa, 2004). O autor ressalta que este último argumento ilumina os demais, pois o conhecimento crítico da relevância sociocultural da matemática pode qualificar as pessoas para intervir nos debates e nas tomadas de decisões sociais que envolvem aplicações da matemática.

\section{Aprendizagem Significativa}

O processo por meio do qual uma nova informação interage com conceitos ou proposições já estabelecidos na estrutura cognitiva do aprendiz é chamado aprendizagem significativa. A interação que caracteriza a aprendizagem significativa transcende uma simples associação. Essa interação ocorre de maneira não-arbitrária e não-literal (substantiva) entre uma nova informação (materiais instrucionais) e as ideias já estabelecidas na estrutura de conhecimentos do aprendiz.

A relação não-arbitrária significa que a nova informação não se relacionará com qualquer aspecto da estrutura cognitiva, mas, sim, com aspectos específicos e relevantes para a assimilação da nova informação em questão. Já a relação não-literal quer dizer que o conhecimento resultante da interação entre o novo e o prévio não será exatamente aquele registrado nos manuais, pois incluirá experiências individuais e reações atitudinais idiossincráticas e afetivas, resultando em significados singulares.

Mediante a interação entre o novo e o prévio, há um compartilhamento de significados: a nova informação passa a ter sentido para o aprendiz e os conhecimentos estabelecidos ficam cada vez mais elaborados, mais estáveis e diferenciados, mais capazes de servirem como âncora em novas interações, o que contribui para o desenvolvimento da estrutura cognitiva. Por esse motivo, o conhecimento, ou mais amplamente a estrutura cognitiva (estrutura de conhecimentos e suas inter-relações), é produto da aprendizagem significativa por definição (Ausubel, 2003).

Em distinção à aprendizagem significativa, Ausubel et al. (1980) definem aquilo que chamam aprendizagem mecânica. Nesse tipo de aprendizagem, a nova informação estabelece uma ligação simples, que é arbitrária e não substantiva, com elementos preexistentes da estrutura cognitiva. A nova informação não se incorpora ao repertório cognitivo do aprendiz, nem o modifica, pois não há interação entre o novo e algum aspecto específico e relevante da estrutura cognitiva já existente. Como o aprendiz não dá significados ao que aprende, o produto da aprendizagem mecânica é, geralmente, de duração, utilidade e significados transitórios.

Existem duas condições para a ocorrência da aprendizagem significativa (Ausubel et al., 1980):

1. o material instrucional deve ser potencialmente significativo;

2. o aluno deve ter disposição para aprender significativamente.

Um material de aprendizagem satisfaz à condição (1) quando possui significado lógico e significado psicológico (real ou fenomenológico). O significado lógico refere-se à natureza própria do material. A evidência desse significado reside na possibilidade de relacionamento não-arbitrário e nãoliteral do material de aprendizagem a uma estrutura cognitiva apropriada e relevante. Ou seja, o material instrucional deve se situar dentro das possibilidades intelectuais do aprendiz naquele momento.

Já o significado psicológico concerne à natureza da estrutura cognitiva. A evidência desse significado repousa na disponibilidade de conhecimentos prévios adequados para a ancoragem do novo material. Em síntese, um material de aprendizagem potencialmente significativo "[...] deve ser relacionável à estrutura cognitiva e o aprendiz deve ter o conhecimento prévio necessário para fazer esse relacionamento de forma não-arbitrária e não-literal" (Moreira, 2012, p.36). A interação não-arbitrária e não-literal entre o conteúdo logicamente significativo do material de aprendizagem e os conceitos específicos e relevantes preexistentes na estrutura de conhecimentos provocam o surgimento do significado real para o aprendiz, que é de natureza idiossincrática.

Todo material instrucional será sempre "potencialmente significativo", nunca significativo, pois a significatividade do material não depende do material em si, mas do sujeito que aprende. Ou seja, " [...] não existe livro significativo, nem aula significativa, nem problema significativo..., pois o significado está nas pessoas, não nos materiais" (Moreira, 2012, p.36). Certamente, isso nos leva à condição (2): ter disposição para aprender significativamente.

Essa condição exige que o aluno tenha de manifestar uma intencionalidade para relacionar, de maneira não-arbitrária e não-literal, o novo material à sua estrutura de conhecimentos. Caso contrário, se for do interesse do aprendiz apenas memorizar o conteúdo para obter os escores necessários para a aprovação, não importa quão potencialmente significativo seja o material, “[...] tanto o processo de aprendizagem como o produto da aprendizagem serão automáticos" (Ausubel et al., 1980, p.34).

Não se trata exatamente de motivação, ou de gostar da matéria. Por alguma razão, o sujeito que aprende deve se predispor a relacionar (diferenciando e integrando) interativamente os novos conhecimentos a sua estrutura cognitiva prévia, modificando-a, enriquecendo-a, 
elaborando-a e dando significados a esses conhecimentos. Pode ser simplesmente porque ela ou ele sabe que sem compreensão não terá bons resultados nas avaliações. (Moreira, 2012, p.37).

Em resumo, para que um conteúdo matemático seja aprendido significativamente, é necessário: (1) que o material instrucional seja adequado às limitações e potencialidades do nível intelectual do aprendiz, (2) que o aluno tenha conhecimentos prévios, especificamente relevantes para a ancoragem do novo material, e (3) que o aprendiz desenvolva uma atitude de relacionar, de maneira não-arbitrária e não-literal, as novas informações a aspectos específicos e relevantes de sua estrutura cognitiva.

\section{Modelagem Matemática e Aprendizagem Significativa}

Embora haja, na literatura especializada, várias discussões acerca de temas que envolvem o caráter didático-pedagógico da modelagem matemática, sendo que algumas visões teóricas se complementam e outras apontam em direções variadas, não há dúvidas quanto à centralidade de dois elementos, fundamentais para o desenvolvimento de atividades de modelagem: (1) o interesse pela investigação de um tema e (2) os problemas com referência na realidade (problemas da vida real). Esses elementos podem ser colocados em paralelo às condições para a ocorrência da aprendizagem significativa. Problemas com referência na realidade ao lado da primeira condição: o material instrucional deve ser potencialmente significativo. E a investigação de um tema de interesse dos alunos ao lado da segunda condição: o aluno deve ter predisposição para aprender significativamente.

Acreditamos que, por ter referência em contextos socioculturais familiares aos alunos, os temas e problemas de modelagem colocados possuem significado lógico. Isso porque permitem o estabelecimento de uma relação nãoarbitrária e não-literal com ideias correspondentemente relevantes, situadas no domínio da capacidade intelectual dos educandos. Assim, eles constituem situações inteligíveis por sua familiaridade sociocultural. Pela mesma razão, tais temas e problemas apresentam maior possibilidade de o aprendiz ter conhecimentos prévios que possam ser utilizados para dar significado real às atividades. Dessa forma, as atividades de modelagem matemática podem ser caracterizadas como potencialmente significativas. Tem-se, portanto, o cumprimento da condição (1).

A motivação oriunda do interesse pela investigação de um tema pode conduzir ao cumprimento da condição (2). Esse interesse pode engajar o aprendiz na resolução da questão formulada, levando-o a assumir uma postura ativa na construção do conhecimento. Por isso, ele pode manifestar a atitude de não apenas memorizar, mas, sobretudo, de adquirir significados. Cabe ressaltar que as variáveis motivacionais e atitudinais estimulam e apressam o processo de interação cognitiva, resultando na emergência de significados por aumentar o esforço, a atenção e a prontidão imediata para a aprendizagem (Ausubel, 2003).

Pode-se afirmar que, como sugerem os parágrafos supracitados, o ambiente de aprendizagem, suscitado pela modelagem matemática, é capaz de favorecer o relacionamento não-arbitrário e não-literal dos conteúdos matemáticos, abordados nessas atividades, a ideias específicas e relevantes, preexistentes na estrutura cognitiva do aluno. Uma compreensão genuína, produto de uma aprendizagem significativa, implica o domínio de significados claros, precisos, diferenciados e transferíveis. A maneira mais viável de testar se os educandos compreenderam significativamente as ideias que são capazes de verbalizar é por meio da solução criativa de problemas (Ausubel et al., 1980).

Há duas maneiras fundamentais de se abordar um problema (Ausubel et al., 1980): a primeira delas é a abordagem por tentativa e erro, que consiste em testar de forma aleatória ou sistemática as alternativas disponíveis, fazendo correções e buscando aproximações até que uma variante bem-sucedida emerja; a segunda é a abordagem pelo discernimento, que implica uma disposição orientada para a formulação e comprovação de hipóteses, com o objetivo de compreender as relações de causa e efeito, subjacentes à situação-problema.

A solução de problemas é um método válido e prático para se buscar evidências de aprendizagem significativa quando os problemas colocados propiciam uma abordagem pelo discernimento. Embora a abordagem por tentativa e erro tenha certo valor pedagógico, esse processo, assim como o uso acrítico de algoritmos, pode conduzir a uma solução bem-sucedida sem qualquer compreensão genuína da razão pela qual a solução é correta. Isso privilegia um ambiente de aprendizagem mecânica.

Visto que a abordagem a ser empregada depende muito do tipo de problema envolvido, faz-se necessário propor situações que exijam ir além da informação adquirida; que favoreçam a interpretação, a formulação e comprovação de hipóteses, a elaboração de variadas estratégias de solução, a verbalização dos resultados e o uso produtivo do erro. Esses problemas devem ser apresentados em contextos variados, formulados de maneira nova e não familiar, de tal forma que requeiram máxima transformação do conhecimento adquirido (Ausubel et al., 1980; Ausubel, 2003).

O ambiente de investigação e problematização, no qual se baseia a modelagem, exige que os aprendizes mobilizem tanto as ideias e habilidades matemáticas previamente aprendidas, quanto os componentes da situação problemática em questão para atingir determinado objetivo, e isto requer a predominância da abordagem pelo discernimento. Esses conceitos e procedimentos matemáticos são inseridos em contextos que sugerem uma gama de possibilidades didáticopedagógicas, envolvendo até mesmo situações aparentemente não matemáticas. Ao contemplarem as etapas de escolha do tema, de formulação de questões de pesquisa, de coleta de dados, de resolução de problemas mediante a construção criativa de modelos matemáticos e de análise crítica dos 
resultados, os aprendizes são levados a transformar ao máximo o conhecimento adquirido. Em outras palavras, um bom desempenho em atividades de modelagem matemática fornece uma forte evidência de que o aluno está aprendendo significativamente os conteúdos matemáticos em questão.

\section{Conclusão}

A modelagem matemática auxilia o professor no cumprimento do seu trabalho, que consiste em personalizar, temporalizar e contextualizar os conteúdos matemáticos. Não se trata apenas de comunicar um conhecimento ao aprendiz em sua forma pronta e acabada.

Em atividades de modelagem matemática, a responsabilidade pela construção do conhecimento é compartilhada com os alunos, tornando-os sujeitos ativos no processo de ensino e aprendizagem dos conteúdos abordados. Tal fato contrapõe-se ao ensino tradicional da matemática, no qual o professor encontra-se no centro do processo educacional e sua atuação resume-se, geralmente, na apresentação precoce de resultados gerais, envolvendo conteúdos formalizados.

Por outro lado, as atividades de modelagem matemática permitem aos alunos vivenciarem alguns passos dados por aqueles que produzem conhecimento, como as inúmeras reflexões, as tentativas infrutíferas e as mudanças de percurso. Essas vivências só podem ser exploradas, no sentido de favorecer a construção de um conhecimento crítico dos conteúdos matemáticos, em ambientes de problematização e investigação que extrapolam o contexto matemático, daí a insuficiência da abordagem definição-exemplo-exercício.

Assim sendo, o processo que envolve a resolução de problemas da realidade mediante a construção, aplicação e avaliação de modelos matemáticos contribui para o ensino da matemática com uma alternativa metodológica que, além de tornar o saber matemático acessível e desejável aos educandos, ainda fornece uma multiplicidade de situações que lhes exigem ir além da informação adquirida - situações que inibem a aprendizagem mecânica e favorecem a significativa.

\section{Referências}

Almeida, L. M.W., \& Dias, M.R. (2004). Um estudo sobre o uso da modelagem matemática como estratégia de ensino e aprendizagem. Bolema, 17(22), 19-36.

Ausubel, D.P., Novak, J.D., \& Hanesian, H. (1980). Psicologia educacional. Rio de Janeiro: Interamericana.
Ausubel, D.P. (2003). Aquisição e retenção de conhecimentos: Uma perspectiva cognitiva. Lisboa: Plátano Edições Técnicas.

Barbosa, J. C. (2001). Modelagem na Educação Matemática: Contribuições para o debate teórico. In Anais da $24^{a}$ Reunião Anual da ANPEd. Caxambu, MG: Associação Nacional de Pós-Graduação e Pesquisa em Educação. http://24reuniao. anped.org.br/tp1.htm\#gt19.

Barbosa, J. C. (2004). Modelagem matemática: O que é? Por quê? Como? Veritati, (4), 73-80.

Bassanezi, R.C. (2010). Ensino-aprendizagem com modelagem matemática: Uma nova estratégia. São Paulo: Contexto.

Biembengut, M.S., \& Hein. N. (2014). Modelagem Matemática no ensino. São Paulo: Contexto.

Borssoi, A.H., \& Almeida, L.M.W. (2004). Modelagem matemática e aprendizagem significativa: uma proposta para o estudo de equações diferenciais ordinárias. Educação Matemática Pesquisa, 6(2), 91-121.

Burak, D. (2004). Modelagem matemática e a sala de aula. In Anais do $1^{\circ}$ Encontro Paranaense de Modelagem em Educação Matemática. Londrina, PR: Universidade Estadual de Londrina.

Burak, D., \& Barbieri, D. D. (2005). Modelagem Matemática e suas implicações para a Aprendizagem Significativa. In Anais da $4^{a}$ Conferência Nacional Sobre Modelagem e Educação Matemática. Feira de Santana, BA: Universidade Estadual de Feira de Santana.

Carvalho, J. P. (1994). Avaliação e perspectivas da área de ensino de matemática no Brasil. Em Aberto, 14, (62), 74-88.

D’Ambrósio, U. (1986). Da realidade à ação: Reflexões sobre educação e matemática. São Paulo: Summus.

Gil, A. C. (2008). Métodos e técnicas de pesquisa social. São Paulo: Atlas.

Lima, T.C.S., \& Mioto, R.C.T. (2007). Procedimentos metodológicos na construção do conhecimento científico: a pesquisa bibliográfica. Revista Katálysis, 10, 37-45.

Marconi, M.A., \& Lakatos, E.M. (2003). Fundamentos de metodologia científica. São Paulo: Atlas.

Moreira, M.A. (2012). ¿Al final, qué es aprendizaje significativo? Revista Qurriculum, (25), 29-56.

Niss, M. (2012). Models and modelling in mathematics education. EMS Newsletter, 49-52.

Santos, L.M.M., \& Bisognin, V. (2007). Experiências de ensino por meio da modelagem matemática na educação fundamental. In J.C. Barbosa, A.D. Caldeira \& J.L. Araújo. Modelagem Matemática na Educação Matemática brasileira: Pesquisas e práticas educacionais. (pp. 99-114). Recife: SBEM. 